\title{
Cutaneous leishmaniasis in Moroccan children: about 12 cases
}

\section{Introduction}

Cutaneous leishmaniasis (CL) is an infectious disease making a real public health problem in some countries such as Morocco. Leishmania major has a high incidence rate in all North Africa countries where it is considered as a public health threat. Zoonotic CL (ZCL) is distributed in the arid and Saharan bioclimatic stages where it follows an epidemic pattern with seasonal occurrence of cases. Zoonotic CL incidence and the interval between epidemics depend on climatic factors. ${ }^{1}$ The purpose of our work was to determine the particularities of child's CL.

\section{Leishmaniasis life cycle}

During the blood meal in the exposed areas of human body, female sand flies injects promastigotes in their infective stage into the skin; The parasites are captured by phagocytes in dermal tissue, initially by short-lived neutrophils (the first leukocyte recruited to the bite site that subsequently enter into apoptosis) and subsequently also by macrophages. In addition to phagocytized parasites, macrophages also phagocytize infected apoptotic neutrophils. Inside the macrophages, promastigotes establish an intracellular residence and transform into aflagellate amastigotes, where they multiply, and are released upon cell lysis, when too many amastigotes are present in the phagolysosome, resulting in reinfection of other cells. The cycle is completed when other sandfly ingest infected phagocytes during the blood meal. Inside the vectors, amastigotes are converted into promastigotes in hindgut/midgut. At this site, parasites proliferate and differentiate into infective promastigotes and migrate to the salivary glands of the sand fly and perpetuate its life cycle.

\section{Material and methods}

This is a retro-prospective single center study of all cases of childhood CL, observed in the dermatology department of the Hassan II hospital in Fez between 2014 and 2016. The diagnosis

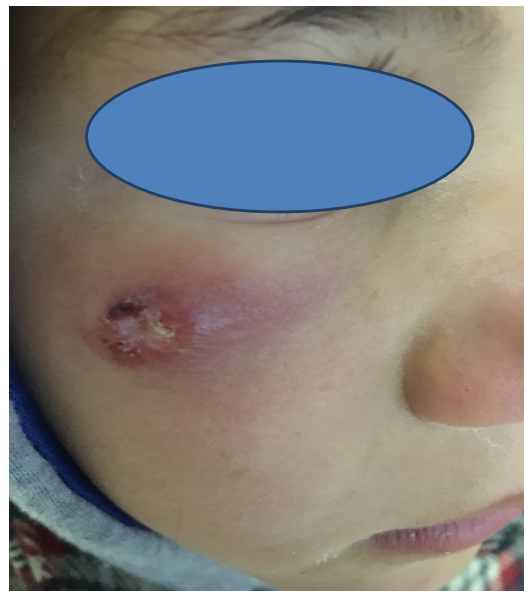

Figure I Erythemato-ulcerative patch of the cheek.
Volume 8 Issue 3 - 2018

\section{Aicha Nassiri, Niema Aqil, Hanane Baybay, Fatima zahra Mernissi}

Department of dermatology, Hospital Center University Hassan li, Morocco

Correspondence: Aicha Nassiri, Department of dermatology, Hospital Center University Hassan li , hassan II hospital, road of sidi hrazem, FES, Morocco, Email aichanassiri6@gmail.com

Received: March 01, 2018| Published: June 08, 2018

of CL was retained on a set of epidemiological-clinical arguments, on the positivity of the direct parasitological examination and/or an evocative histopathological aspect.

\section{Results}

Twelve children were collected. The average age was 11.3 years with a minimum age of 1 year and a sex-ratio $\mathrm{H} / \mathrm{F}$ of 0.93 . All patients lived or had been in endemic areas. A fall preponderance was noted $(41.6 \%)$. The most common clinical aspect was ulcero-crusted nodule $(53.3 \%)$, single $(63.3 \%)$ and on the face $(65 \%)$. One patient presented mucocutaneous leishmaniasis. The diagnosis was clinically suspected and confirmed by direct parasitological examination $(56.6 \%)$ or histological examination (35\%). Glucantime ${ }^{\circledR}$ was the treatment of choice, used intralesionally (70\%), cryotherapy and blue light were adjuvant therapy in half of the patients. The evolution was favorable in almost all the cases in a variable delay of 4 weeks to 8 weeks. The use of systemic treatment was necessary in 2 children: glucantime in IM, in one patient it was complemented with dynamic phototherapy. Scars were noted in 2 cases (18\%) with residual pigmented ( 1 case) or atrophic (1 case) scars. (Table 1), (Figures 1-5).

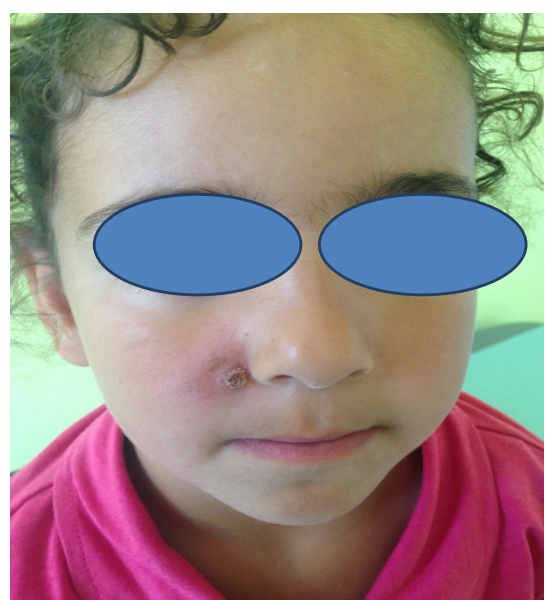

Figure 2 Crusty papule of the cheek. 


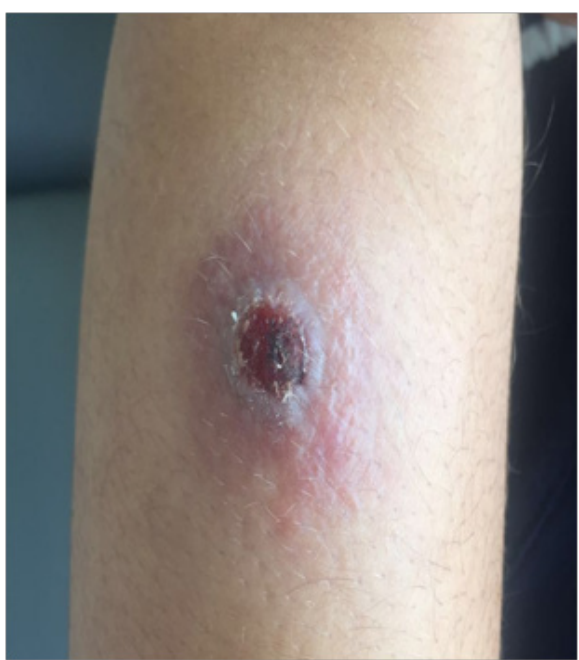

Figure 3 ulcerative nodule forearms.

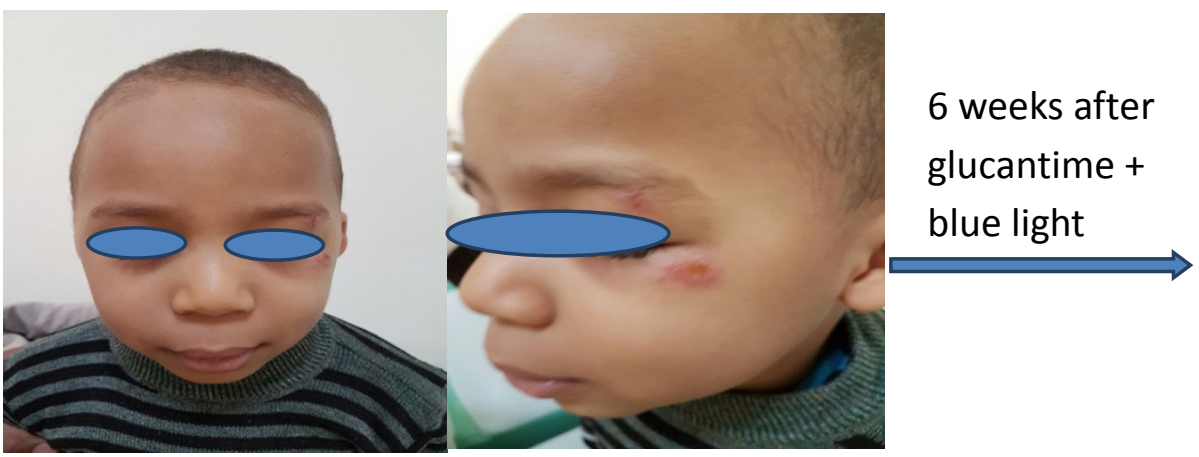

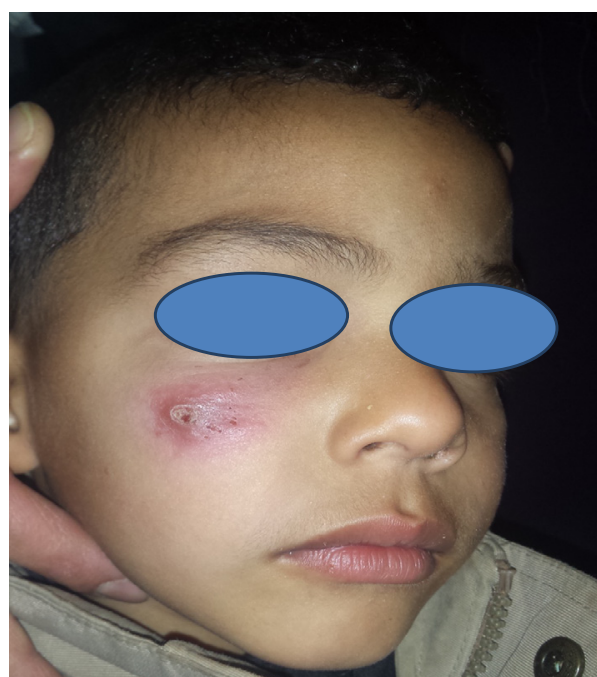

Figure 4 Ulcerative nodule of cheek.

Figure 5 Ulcerative nodule of the cheek.

Table I Patients presentation, treatment and out come

\begin{tabular}{lllll}
\hline Patients & Age (Years & Treatment & & Out Come \\
\hline I & II & Ulcero crusted nodule & Intralesionnal glucantime & Complete Healing \\
2 & I5 & Ulcero crusted nodule & Intralesionnal glucantime & Atrophic scar \\
3 & 10 & Erosive papule & Glucantime in IM & Complete Healing \\
4 & 8 & Ulcero crusted nodule & Glucantime in IM & Complete Healing \\
5 & 14 & Ulcero crusted nodule & Intralesionnal glucantime & Complete Healing \\
6 & 12 & Erosive papule & Intralesionnal glucantime & Complete Healing \\
7 & 14 & Ulcerative nodule & Intralesionnal glucantime & Complete Healing \\
8 & 15 & Ulcero crusted nodule & Intralesionnal glucantime & Complete Healing \\
9 & 1 & plaque & Intralesionnal glucantime & Complete Healing \\
10 & 13 & Ulcerative nodule & clarithromycine & Complete Healing \\
11 & 16 & Ulcero crusted nodule & clarithromycine & Pigmented scar \\
\hline 12 & 6 & plaque & Intralesionnal glucantime & Complete Healing \\
\hline
\end{tabular}

\section{Discussion}

Our serie is characterized by a predominance of women, which ties in with Tunisian data. ${ }^{1,2}$ The average consultation time, relatively long in our series, could be explained mainly by the painless ness of the lesions. To our knowledge, there are only a few series published in the literature on cutaneous leishmaniasis in children. The average age of patients was lower than that of patients with a series of Algeria and higher than that of patients with a Tunisian series. ${ }^{3,2}$ Most of the skin lesions in our series were ulcerated. Many clinical presentations are possible: papulo-nodular, lupoidal, sporotrichoid, impetigo and erysipeloid. ${ }^{4}$ The lesions are often unique and localized very 
frequently in the face, because this location is easily accessible to sandflies. ${ }^{5,6}$ Our series is characterized by frequent participation of the hands and forearms, while in another moroccan series lesions were predominant on the cheeks. ${ }^{2}$ To confirm the diagnosis, it is necessary to carry out a parasitological examination. The story may reveal a family history of cutaneous leishmaniasis in 5 percent of cases as the case of $12 \%$ of our patients. ${ }^{5}$ Therapeutic abstention, when it finds its indication, is recommended more broadly in children because of the binding nature of the treatment. ${ }^{7}$ Meglumine antimonite intralesional is the treatment of choice for localized lesions. ${ }^{5}$ This treatment is effective and well tolerated in children. Intramuscular injections of meglumine antimonite can be used in case of periorificiel involvement, multiple lesions, a poor response to local treatment, satellite lesions testifying to cutaneous or lymphatic spread, and in the case of regional lymphadenopathy. Other treatments proposed in cutaneous leishmaniasis in children such as Clarithromycin, fluconazole, dapsone, azithromycin and rifampicin. Surgery, cryotherapy, laser and intralesional bleomycin have been used successfully. These treatments have a very limited use because they are not subject to rhandomized evaluations. ${ }^{3,2,7}$

\section{Conclusion}

CL is not un common in the north Afrrican Saharan area. It is relatively common in children especially during the second decade. It is characterized by its usual favorable evolution and the absence of clinical particularities compared to the adult. Treatment modalities should be taylored on a case by case basis.

\section{Acknowledgments}

I hereby acknowledge and agree that I, aicha nassiri, and the principal author of the manuscript cutaneous leishmaniasis in Moroccan children: about 12 cases.

\section{Conflict of interest}

The authors declared there is no conflict of interest.

\section{References}

1. Toumi A, Chlif S, Bettaieb J, et al. Temporal dynamics and impact of climate factors on the incidence of Zoonotic cutaneous leishmaniasis in central Tunisia. PLoS Neg1 Trop Dis. 2012;6(5):e1633.

2. Fennich S, Souissi A, benmously R, et al. Childhood cutaneous leishmaniasis in Tunisia : retrospective study of 60 cases. Med Trop (Mars). 2006;66(5):456-460.

3. Khelil A, Serradj A, Belkorissat F, et al. Profil epidemiologique de la leishmaniose cutanée de l'enfant (26 cas). Ann Dermatol venereol. 2003;130(4):53.

4. Qasmi S, Elguelbazouri N, Belgnaoui FZ, et al. Childhood cutaneous leishmaniasis: Experience of a Moroccan unit of dermatology. Dermatol Online J. 2008;15;14(12):18.

5. Rhajaoui M, Nasereddin A, Fellah H, et al. New clinico-epidemiologic profile of cutaneous leishmaniasis, Morocco. Emerg Infect Dis. 2007;13(9):1358-1360.

6. kharfi M, Benmously R, El Fekih N, et al. Childhood leishmaniasis, Report f 106 cases. Dermatol Online J. 2004;10(2):6.

7. Talaari SA, Talaei R, Shajari G, et al. Taghaviardakani A. Childhood cutaneous leishmaniasis, report of 117 cases from Iran. Korean $J$ Parasitol. 2006;44(4):355-360. 